\title{
THE INFLUENCE OF THE RADIATION PRE-TREATMENT ON ELECTRICAL PROPERTIES OF MINERALS
}

\author{
IVAN FLOREK AND INGRID MUROVÁ \\ Institute of Geotechnics of the Slovak Academy of Sciences, Watsonova 45, \\ Košice, Slovakia
}

(Received in the final form August 15, 1995)

\begin{abstract}
The radiation pre-treatment process makes possible the mineral properties modifications. In this paper theoretical principles of the influence of the radiation pre-treatment by means of accelerated electrons on the electrical conductivity and permittivity of selected minerals is analysed. The changes of specific conductivity, as well as permittivity in dependence on the radiation dose and the grain size of minerals are introduced. On the basis of these data the conditions of the above mentioned physical quantities changes using the radiation pre-treatment from the mineral processing improvement point of view are defined.
\end{abstract}

\section{INTRODUCTION}

The pre-treatment of minerals by a high-energy radiation represents a new and progressive treatment method which enables to modify particularly the physical and physico-chemical properties of various minerals, and in this way to ensure a high efficiency of their further processing. For this purpose, the radiation by accelerated electrons produced in various types of accelerators, is most frequently used in the industry. From the operational point of view, it is more advantageous than alternative radionuclide radiation. So far it was applied in the mining industry on a laboratory scale only, for the modification of magnetic and flotation properties of industrial minerals [1 to 3 ].

The energy of the accelerated electrons applied in the process of the radiation pre-treatment of minerals affects substantially the electric properties of their 
mineral components. This paper provides a summary of the experimental investigations of changes of specific conductivity and permittivity of selected minerals after their irradiation by the accelerated electrons. This effect could be used to increase the efficiency of their electrical separation.

\section{THEORETICAL SECTION}

The electrical properties of minerals, like properties of all crystalline substances, are dependent mainly upon chemical composition, arrangement of basic structural parts and their interactions. These properties are manifested as:

-transport processes related to the displacement of electric charge carriers in the relevant mineral under the influence of the external field, e.g. electrical, magnetic or thermal

-behaviour of the investigated substances in the time-variable electrical field.

Basic parameters which characterise the properties mentioned above and the effects induced by them in minerals are specific conductivity and relative permittivity. From the point of view of conductance, most minerals belong to semiconductors, with their conductivity ranging in a wide interval from $10^{6} \mathrm{Sm}^{-1}$ to $10^{-8} \mathrm{Sm}^{-1}[4]$.

The electrical conductivity of minerals, similarly to that of all solid substances, can be explained by means of the band theory by the motion of electrons in incompletely filled conductivity band of the energy levels of a given crystal. This motion is based on the transition of electrons from one atom to another within the crystal, and is related to their wave properties. Although the last energy band of minerals with semiconducting properties is filled up, a valence band in the close proximity allows, mainly after supplying additional thermal energy, to increase the jumps and motion of the electrons. This is the way by which conductivity of minerals can be increased. 
Permittivity of minerals describes their behaviour in the time-varying electric fields. Its value is characteristic of a mineral. Together with specific conductivity it affects relationship between the density of the induced current and the intensity of the electric field. The relative permittivity of minerals, expressed as a ratio of their permittivity and that of the vacuum, ranges mostly from 2 to 80 SI units.

Characteristic phenomena observed for minerals irradiated by the accelerated electrons include excitation of electrons, ionisation of molecules and their heating accompanied by their possible thermal decomposition. All these phenomena affect significantly the conductivity of the irradiated minerals. The excitation of electrons due to the action of the accelerated electrons accelerates their motion and increases the number of free electrons which is expressed by the growth of the intrinsic conductivity.

Similar effect was observed in the case of ionisation of the molecules of the irradiated minerals which occurs when the quantity of the accelerated electrons of the transmitted energy is greater than the binding energy of a pulled-off electron in the molecule.

The thermal effect of the radiation induced by imparting the energy of the accelerated electrons during their interaction to the mineral accelerates the oscillatory motion of a crystal lattice. This is the way by which free electrons arise and the conductivity of a mineral increases. Possible metal additives in the crystal lattice are also important from the viewpoint. As a result of specific binding proportions and the number of particles in the crystal lattices of individual minerals, the effect of radiation on their specific conductivity and permittivity varies and has to be determined separately for every individual mineral.

\section{EXPERIMENTAL SECTION}

Laboratory investigation of the effect of the irradiation by the accelerated electrons on the above mentioned electrical properties of minerals were carried out on specimens described in Table 1. Their size distribution ranged from 0.1 to $1.0 \mathrm{~mm}$. The irradiation of the samples took place at the linear accelerator of electrons of the type LINAC 4-1200, with the energy range of the accelerated 
Table 1 The origin and the grade of the minerals used in the tests

\begin{tabular}{|c|c|c|c|c|c|c|c|}
\hline Mineral & Location & & Content & \multicolumn{4}{|c|}{ of basic elements $[\%]$} \\
\hline Siderite & Rudňany & & & & 43,06 & & \\
\hline Chalcopyrite & Rudñany & $\mathrm{Cu}$ & 27,07 & $\mathrm{Fe}$ & 31,47 & & 27,82 \\
\hline Pyrite & Hnúšt’a & & & $\mathrm{Fe}$ & 31,33 & & 53,68 \\
\hline Galenite & Banská Štiavnica & $\mathrm{Pb}$ & 83,65 & & & & \\
\hline
\end{tabular}

electrons from 3.5 to $5 \mathrm{MeV}$. Medium current of the beam of the fast electrons was $300 \mu \mathrm{A}$, medium output up to $1.2 \mathrm{~kW}$, and the pulse duration $2.7 \mu \mathrm{s}$. The dose of of the accelerated electrons was controlled by means of the current intensity of the beam of the fast electrons, and by the duration of the irradiation.

The measurements of specific conductivity and of relative permittivity of the selected minerals were carried out by:

-the direct measurement of electrical conductivity by means of semiautomatic bridge Tesla BM 484 [4]

-the capacity measurement of a plate condenser using alternating current of very low frequency for the permittivity determinations [4].

Granular material subjected to the electrical measurements was placed in a holder of a cylindrical shaper, the base surfaces of which were connected to the measuring circuit. Special attention was paid to the conditions of the measurement, particularly to the compression of the specimen, temperature, and to the elimination of the disturbing effects of the force fields [5].

\section{RESULTS AND DISCUSSION}

The experiments proved that the irradiation by accelerated electrons affected the specific conductivity and permittivity of all four investigated minerals (Figures 1 to 4). The observed changes cam be explained by the predominant thermic effect 


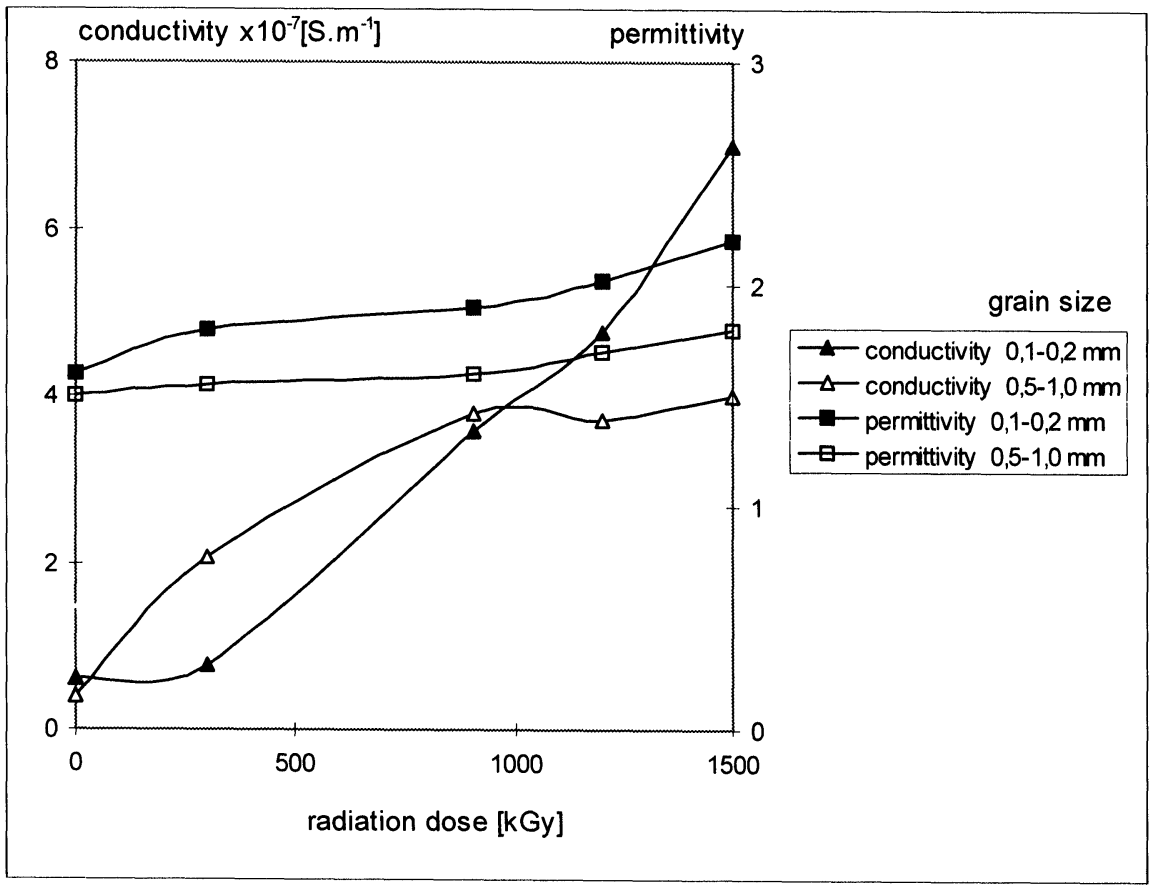

Fig. 1 The effect of irradiation on conductivity and permittivity of siderite

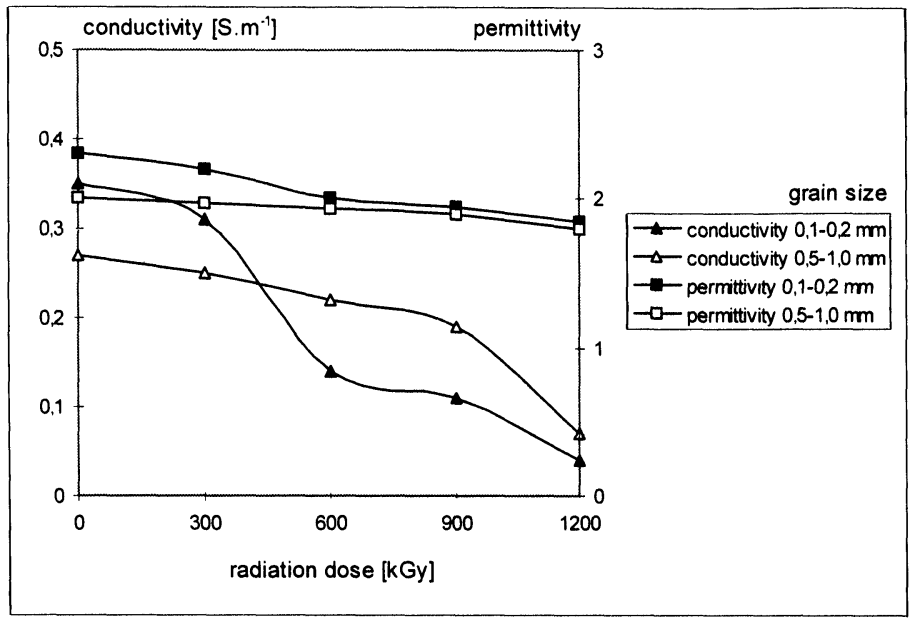

Fig. 2 The effect of irradiation on conductivity and permittivity of chalcopyrite 
of the radiation on the samples of minerals. Processes of the induced excitation and ionisation cease within a fraction of a second after the termination of irradiation [6]. Their influence on the change of the intrinsic and the hole conductivity of minerals cannot be fully excluded. The thermic effect of the radiation incites decomposition of the mineral and formation of new phases with the changed electrical properties, mostly on the surface of its grains.

The warming up of sulphides results in their desulphurisation, starting at a temperature as low as $350^{\circ} \mathrm{C}$ in chalcopyrite and $450^{\circ} \mathrm{C}$ in pyrite [7]. Such a decomposition of sulphides, without the access of the air, and occurring according to the equation

$$
\mathrm{MS}+2 \mathrm{O}_{2} \rightarrow \mathrm{MO}_{2}+\mathrm{SO}_{2}
$$

results in the development of an appropriate metal oxide $\left(\mathrm{MO}_{2}\right)$ in the surface layer of the irradiated mineral grain, the electrical conductivity of which is lower by several orders of magnitude.

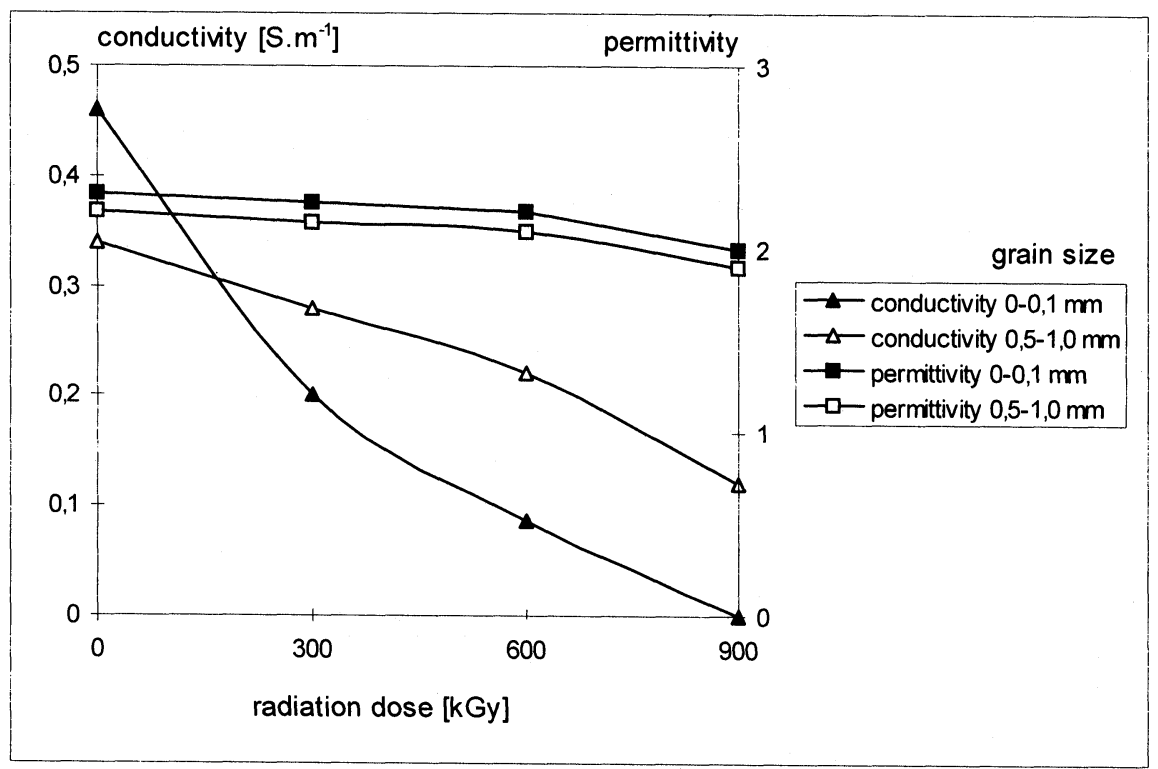

Fig. 3 The effect of irradiation on conductivity and permittivity of pyrite 
For instance, its value is approximately $10^{-1} \mathrm{Sm}^{-1}$ in chalcopyrite and $10^{-2} \mathrm{Sm}^{-1}$ in hematite, arising by irradiation. For this reason, the increase of the irradiation dose results in the decrease of specific conductivity and permittivity in all three investigated sulphides (Figures 2 to 4 ).

In the case of siderite, the irradiation has an opposite effect and results in the improvement of its electrical properties. The irradiation of siderite and the increase of temperature above $400^{\circ} \mathrm{C}$ causes its decarbonisation according to the equation [1]

$$
4 \mathrm{FeCO}_{3}+\mathrm{O}_{2} \rightarrow 2 \mathrm{Fe}_{2} \mathrm{O}_{3}+4 \mathrm{CO}_{2}
$$

According to the irradiation conditions, the developing layer of hematite $\left(\alpha-\mathrm{Fe}_{2} \mathrm{O}_{3}\right)$ or maghemite $\left(\gamma-\mathrm{Fe}_{2} \mathrm{O}_{3}\right)$ exhibits considerably better electrical properties than the original siderite (Fig. 1).

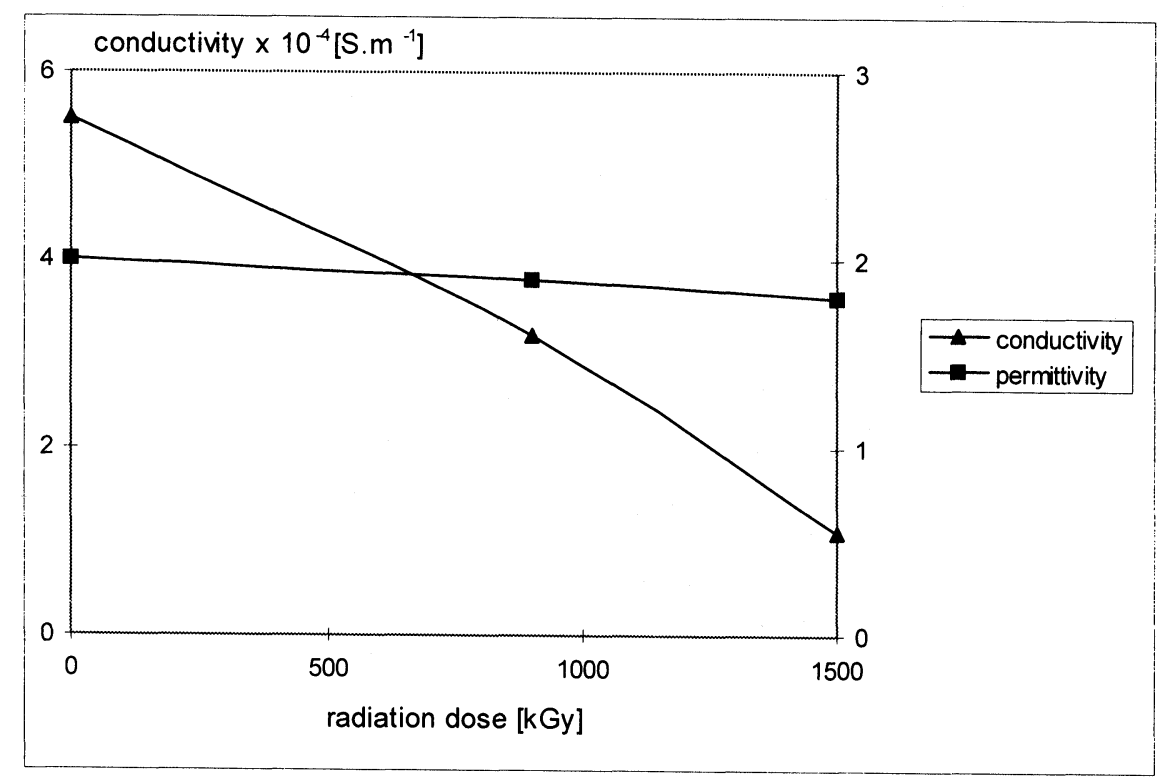

Fig. 4 The effect of irradiation on conductivity and permittivity of galenite (particle size $-0.2+0.1 \mathrm{~mm}$ ). 
The radiation of the accelerated electrons penetrates into the solid substances in proportion to their density and its effect is manifested mainly in their surface layer. To this also corresponds the different course of changes of specific conductivity and permittivity under irradiation of classes of particle sizes of selected minerals (Figures 1 to 4 ). These changes are more pronounced in chalcopyrite and pyrite, decomposition temperature of which is lower than that of galenite and siderite.

The grain size of minerals affects, in particular, the values of their permittivity because the measurement technique used is sensitive to the filling coefficient of the sample holder. Behaviour of the dependence of permittivity on the particle size of the non-irradiated samples of three selected minerals is shown in Figure 5.

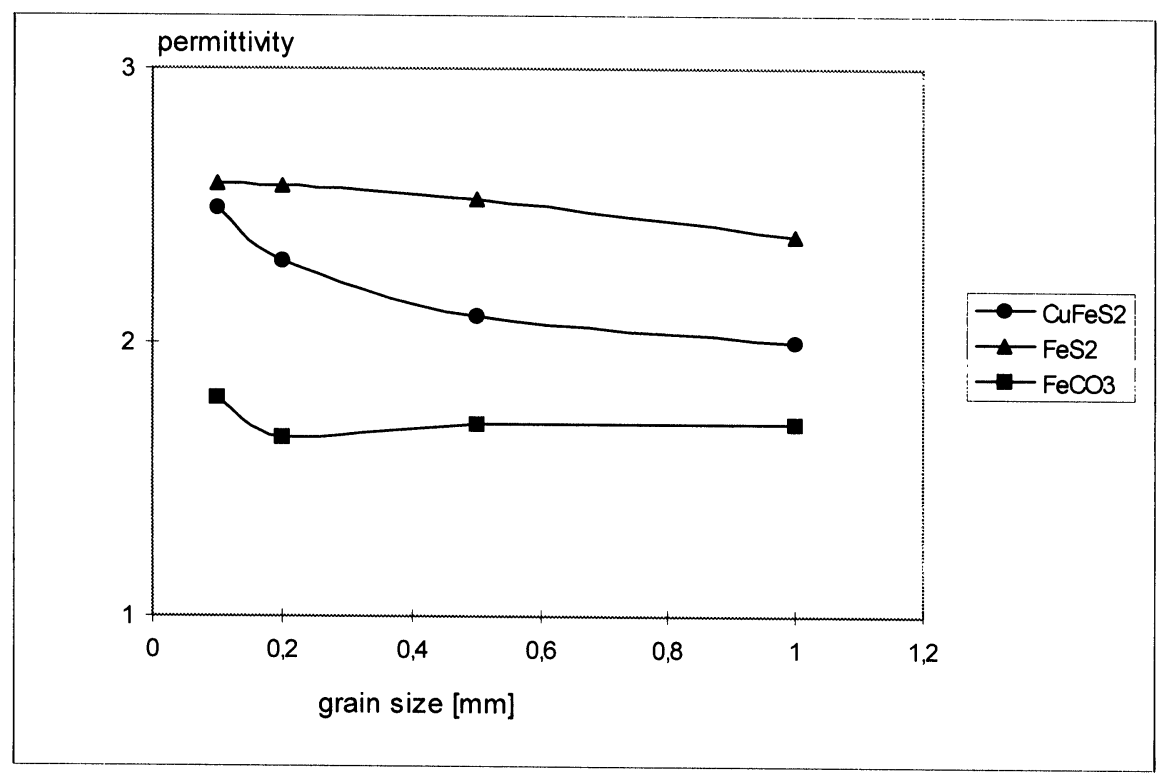

Fig. 5 The relationship between permittivity and particle size of selected materials

This Figure confirms the decrease of the values of permittivity with increasing particle size. The highest decline, by a much as $20 \%$, was exhibited by coarse-grained chalcopyrite, while only $9 \%$ decline was observed in pyrite. The 
mentioned influence of grain size should be taken into consideration also for evaluation of the effect of the radiation of the accelerated electrons on permittivity of irradiated minerals.

Mutual comparison of the magnitude of changes of both investigated electrical quantities of the given mineral, that developed as a result of the irradiation, favours specific conductivity. This fact corresponds to the important influence of the radiation of the accelerated electrons on the surface properties of minerals, also manifested by the changes in specific conductivity. Permittivity expresses the behaviour of a certain volume of a mineral in the electrical field; its change is thus less pronounced.

\section{CONCLUSIONS}

1. Changes of electrical conductivity and permittivity of minerals resulting from collision of the accelerated electrons depend mainly on the magnitude of the irradiation dose and on chemical composition of the minerals.

2. The effect of the above mentioned irradiation on electrical properties of the selected minerals is related, first of all, to its thermic manifestation and decomposition of the relevant mineral induced by this radiation.

3. The radiation of the accelerated electrons affects positively the electrical properties of minerals having a character of dielectrics on the surface of which, under the influence of decomposition, new phases of higher electrical quality develop. On the contrary, this radiation has a negative effect on sulphides exhibiting good electrical properties.

4. A decrease in the particle size and an increase in the specific surface of the irradiated minerals affects positively the process of modification of their electrical properties by irradiation by the accelerated electrons. 


\section{REFERENCES}

[1] I. Florek and I. Murová: Modification of magnetic properties of siderite by radiation pretreatment with accelerated electrons. Fyz. Probl. Metall. 27 (1993), 145

[2] I. Florek: The influence of radiation pretreatment on floatability of tetrahedrite. In: Proc. Intl. Conf. Environment and Mineral Processing, Part II, Ostrava, Czech Republic (1994)

[3] V.I. Rostovcev et al.: Theoretical and practical problems of magnetic separation of minerals (in Russian), DT ČSVTS Košice (1989)

[4] J. Brož et al.: Fundamentals of Physical Measurements (in Czech). SPN Praha (1967)

[5] I. Florek and V. Domaracky: Electric properties of fine grained crystalline substances and their measurement. In: Proc. 3rd Intl. conf. Measurement and Control of Granular Materials, Shenyang, China (1993).

[6] H. Hoberg: Untersuchungen zur Deutung der Anderung der Flotierbarheit halbleitender Erzmineralien durch Bestrahlung im Kernreaktor. Aachener Blatter 17 (1967)

[7] V. Vesely et al.: The roasting of sulphides of zinc, lead, copper and iron. (in Czech). Chem. listy 85 (1991)

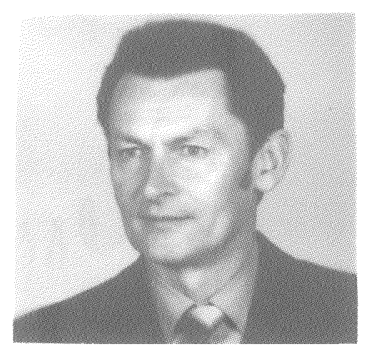

Ivan Florek was born in 1934 and graduated in mineral processing and in economy. He obtained his $\mathrm{PhD}$ degree from the Technical University in Košice. After eight of experience as a head of a milling plant, he joined, in 1969, the former Mining Institute of the Slovak Academy of Sciences. His main research interests include magnetic separation and economy of mineral processing, and recently he included the radiation pretreatment and modification of properties of minerals. Dr. Florek is an author of more than 80 papers and 5 patents and numerous industrial inventions. Currently he is the Research Director of the Institute of Geotechnics.

Ingrid Murova was born in 1970 and graduated with the Dipl. Ing. degree in metallurgy from the Technical University in Košice. At present she is a research assistant at the Institute of Geotechnics of the Slovak Academy of Sciences in Košice.

Keywords: radiation pretreatment, accelerated electrons, permittivity, conductivity, sulphides, carbonates. 